Article

\title{
Genetic Basis and Physiological Effects of Lipid A Hydroxylation in Pseudomonas aeruginosa PAO1
}

\author{
Alessandra Lo Sciuto ${ }^{1, \dagger}{ }^{\dagger}$ Matteo Cervoni ${ }^{1, \dagger}{ }^{+}$, Roberta Stefanelli ${ }^{2}$, Maria Concetta Spinnato ${ }^{1}$, \\ Alessandra Di Giamberardino ${ }^{3}$, Carmine Mancone ${ }^{3}(\mathbb{D})$ and Francesco Imperi ${ }^{1, *(D)}$ \\ 1 Department of Science, Roma Tre University, 00146 Roma, Italy; alessandra.losciuto@uniroma3.it (A.L.S.); \\ matteo.cervoni@uniroma3.it (M.C.); mariaconcetta.spinnato@uniroma3.it (M.C.S.) \\ 2 Department of Biology and Biotechnology Charles Darwin, Sapienza University of Rome, \\ Laboratory affiliated to Istituto Pasteur Italia-Fondazione Cenci Bolognetti, 00185 Roma, Italy; \\ roberta.stefanelli@uniroma1.it \\ 3 Department of Molecular Medicine, Sapienza University of Rome, 00185 Roma, Italy; \\ alessandra.digiamberardino@uniroma1.it (A.D.G.); carmine.mancone@uniroma1.it (C.M.) \\ * Correspondence: francesco.imperi@uniroma3.it \\ $\dagger$ These authors equally contributed to the work.
}

Received: 4 November 2019; Accepted: 8 December 2019; Published: 10 December 2019

\begin{abstract}
Modifications of the lipid A moiety of lipopolysaccharide influence the physicochemical properties of the outer membrane of Gram-negative bacteria. Some bacteria produce lipid A with a single hydroxylated secondary acyl chain. This hydroxylation is catalyzed by the dioxygenase LpxO, and is important for resistance to cationic antimicrobial peptides (e.g., polymyxins), survival in human blood, and pathogenicity in animal models. The lipid A of the human pathogen Pseudomonas aeruginosa can be hydroxylated in both secondary acyl chains, but the genetic basis and physiological role of these hydroxylations are still unknown. Through the generation of single and double deletion mutants in the lpxO1 and lpxO2 homologs of P. aeruginosa PAO1 and lipid A analysis by mass spectrometry, we demonstrate that both LpxO1 and LpxO2 are responsible for lipid A hydroxylation, likely acting on different secondary acyl chains. Lipid A hydroxylation does not appear to affect in vitro growth, cell wall stability, and resistance to human blood or antibiotics in P. aeruginosa. In contrast, it is required for infectivity in the Galleria mellonella infection model, without relevantly affecting in vivo persistence. Overall, these findings suggest a role for lipid A hydroxylation in $P$. aeruginosa virulence that could not be directly related to outer membrane integrity.
\end{abstract}

Keywords: biofilm; Galleria mellonella; hydroxylation; infection; lipid A; LpxO1; LpxO2; resistance; virulence

\section{Introduction}

The cell envelope of diderm (Gram-negative) bacteria consists of two concentric membranes, the cytoplasmic (or inner) and the outer membrane, separated by the periplasmic space. The inner membrane is a typical phospholipids bilayer, while the outer membrane of most diderm bacteria is composed of glycerophospholipids and lipopolysaccharide (LPS) in the inner and outer leaflet, respectively [1]. LPS is a negatively charged glycolipid that, in the presence of divalent cations, forms a tightly packed layer which provides an effective permeability barrier against harmful agents [2].

Pseudomonas aeruginosa is a Gram-negative opportunistic pathogen mostly dreaded for its high antibiotic resistance and for its ability to cause severe infections in immunocompromised patients. It also represents the main cause of chronic lung infection in individuals suffering from cystic fibrosis, bronchiectasis, or chronic obstructive pulmonary disease [3]. P. aeruginosa LPS consists of a hydrophobic lipid A moiety, that anchors the molecule to the outer membrane, a conserved core oligosaccharide 
and a distal O-antigen polysaccharide, that can be present in two chemically distinct types, historically referred to as A-band and B-band $\mathrm{O}$ polysaccharides. B-band $\mathrm{O}$ polysaccharides can vary widely among strains and represent the basis of P. aeruginosa O-serotyping [4]. Both genetics and chemical evidences have confirmed LPS as an essential component of the P. aeruginosa cell envelope [5-7].

P. aeruginosa lipid A consists of an $\mathrm{N}$ - and O-acylated di-glucosamine bisphosphate backbone (Figure 1). While the basic lipid A structure is quite conserved among strains, some heterogeneity is observed in the number of primary acyl groups and the number and nature of secondary acyl groups [4,8]. P. aeruginosa lipid A is mostly present with five or six fatty acid substituents, including two N-linked 12:0 (3-OH) groups at positions 2 and 2' (primary C12 or lauryl chains), one or two O-linked 10:0 (3-OH) groups at positions 3 and/or 3' (primary C10 or decanoyl chains), and two 12:0 groups O-linked to the primary $\mathrm{C} 12$ chains (secondary C12 chains). In most strains, a fraction of lipid A contains an additional secondary 16:0 (palmitoyl) group linked to the primary $\mathrm{C} 10$ chain at position $3^{\prime}$ (Figure 1) [4,8].

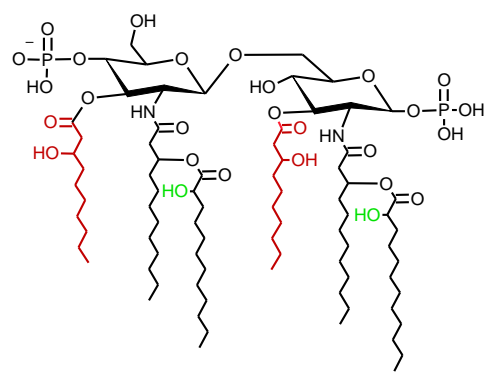

MW: 1633

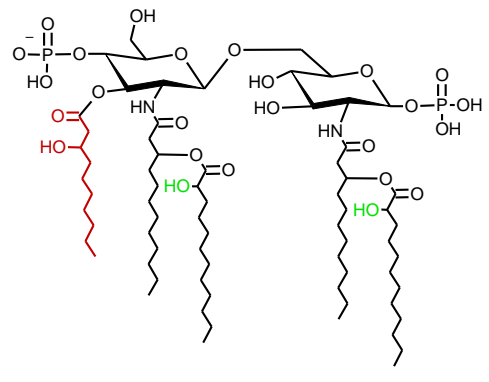

MW: 1463

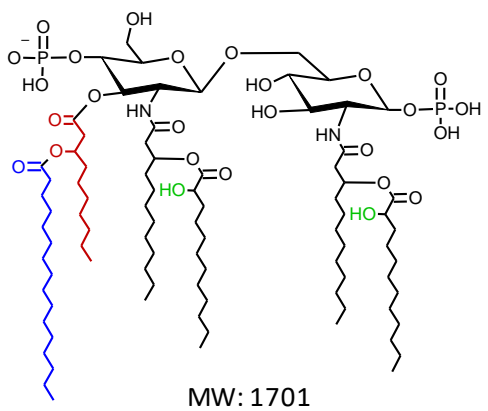

MW: 1701

Figure 1. Chemical structure and molecular weight (MW) of the lipid A species most commonly found in P. aeruginosa, i.e., the hexa-acylated lipid A (left), the penta-acylated lipid A (middle), and the penta-acylated lipid A with a secondary C16 (palmitoyl) chain (right). The primary and secondary C12 acyl chains are in black, the primary C10 chains are in red, and the palmitoyl chain is in blue. The predicted hydroxyl groups of the secondary $\mathrm{C} 12$ acyl chains are highlighted in green. Chemical structures were drawn with the ACD/ChemSketch software, which also calculated the MW.

P. aeruginosa lipid A from different strains and culture conditions also show a variable degree of hydroxylation of the secondary $\mathrm{C} 12$ chains $[4,8-10]$. In most of the Gram-negative bacteria that produce lipid A with hydroxylated secondary acyl chains, the incorporation of the hydroxyl group in lipid A fatty acids is catalyzed by the alpha-ketoglutarate-dependent dioxygenase LpxO, first characterized in Salmonella [11,12]. In Klebsiella pneumoniae and Acinetobacter baumannii, lipid A hydroxylation increases resistance to cationic antimicrobial peptides (e.g., polymyxins) and is required for full virulence, as demonstrated in the insect Galleria mellonella infection model [13,14]. Lipid A hydroxylation was also found to be important for Burkholderia pseudomallei replication within macrophages [15], and for K. pneumoniae persistence in the lung of experimentally infected mice [16].

Interestingly, while all LpxO-proficient Gram-negative bacteria investigated so far have a single hydroxylated secondary acyl chain in lipid A [12-15], the lipid A of P. aeruginosa can be hydroxylated in both secondary $\mathrm{C} 12$ chains, and two lpxO orthologs have been identified in P. aeruginosa genomes, named lpxO1 and lpxO2 [4,8,10] (www.pseudomonas.com). However, the involvement of these two genes in lipid A hydroxylation, as well as the role of this lipid A modification in P. aeruginosa physiology, has not been experimentally investigated thus far.

In this work, we used reverse genetics and mass spectrometry to confirm that both lpxO1 and $l p x \mathrm{O} 2$ are responsible for lipid A hydroxylation, likely acting on different $\mathrm{C} 12$ chains. We also demonstrate that, differently from other Gram-negative bacteria, lipid A hydroxylation has no relevant effects on P. aeruginosa PAO1 growth, cell envelope integrity, and antibiotic and human blood resistance, although it is required for pathogenicity in the G. mellonella model of acute infection. 


\section{Materials and Methods}

\subsection{Bacterial Strains and Growth Conditions}

Bacterial strains used in this study are listed in Table S1. Bacteria were cultured in Lysogeny Broth, Lennox formulation (Acumedia) for genetic manipulation, while growth, biofilm, and antibiotic resistance assays were performed in Mueller-Hinton broth (MH; Difco) or M9 minimal medium [17] supplemented with $50 \mu \mathrm{M} \mathrm{FeCl}_{3}$ and $20 \mathrm{mM}$ succinate as the carbon source. Planktonic growth assays were performed in 96-well microtiter plates ( $200 \mu \mathrm{L}$ of medium in each well) at 22,30 , or $37^{\circ} \mathrm{C}$ and the optical density at $600 \mathrm{~nm}\left(\mathrm{OD}_{600}\right)$ of bacterial cultures was measured in a Tecan Spark 10M microtiter reader. When required, antibiotics were added for the selection of recombinant strains on agar plates at the following concentration for E. coli (the concentration used for P. aeruginosa is shown between brackets): nalidixic acid $20 \mu \mathrm{g} / \mathrm{mL}$, chloramphenicol $30 \mu \mathrm{g} / \mathrm{mL}(350 \mu \mathrm{g} / \mathrm{mL})$.

\subsection{Generation of Deletion Mutants}

Unmarked in-frame deletion mutants in $l p x O 1$ and $l p x O 2$ genes were obtained using the sacB-based suicide plasmid pDM4 [18] (Table S1). E. coli was used for recombinant DNA manipulations. The constructs for mutagenesis were generated by directional cloning into pBluescript II (Stratagene) two DNA fragments of ca. $500 \mathrm{bp}$, encompassing the regions upstream and downstream of the gene to be deleted. All DNA fragments were amplified by PCR with Q5 Hot Start High-Fidelity DNA Polymerase (New England Biolabs) using the genomic DNA of P. aeruginosa PAO1 as the template. Primers and restriction enzymes used for cloning are described in Table S2. After DNA sequencing checks, the upstream and downstream fragments were excised from pBluescript II and sub-cloned into pDM4, yielding $\mathrm{pDM} 4 \Delta l p x \mathrm{O} 1$ and $\mathrm{pDM} 4 \Delta l p x \mathrm{O} 2$ (Table S1). The pDM4 derivatives were conjugally transferred from E. coli S17.1 $\lambda$ pir into P. aeruginosa, and in-frame deletion mutations were obtained by recombination and sucrose-based selection as described [19]. Gene deletions in P. aeruginosa were verified by PCR and DNA sequencing.

\subsection{Generation of Plasmids}

The pMElpxO1 and pMElpxO2 constructs were generated by PCR amplification and directional cloning of the DNA fragment encompassing the coding sequence of each gene into the isopropyl-1-thio-ßD-galactopyranoside (IPTG) inducible shuttle vector pME6032 [20], using the primers and restriction enzymes reported in Table S2. The constructs were verified by DNA sequencing and introduced in $P$. aeruginosa strains by transformation using chemically competent cells.

\subsection{Lipid A Analysis}

Lipid A was extracted from bacterial cell pellets using the ammonium hydroxide-isobutyric acid-based procedure as previously described [10,21]. Briefly, cells from $2 \mathrm{~mL}$ of early stationary phase cultures in MH were collected by centrifugation and resuspended in $400 \mu \mathrm{L}$ of $70 \%(\mathrm{v} / \mathrm{v})$ isobutyric acid and $1 \mathrm{M}$ ammonium hydroxide (5:3). After 1-h incubation at $100{ }^{\circ} \mathrm{C}$ and centrifugation at $2000 \times g$ for $15 \mathrm{~min}$, supernatants were added to $400 \mu \mathrm{L}$ of endotoxin-free water, frozen at $-80^{\circ} \mathrm{C}$, and lyophilized in a centrifugal vacuum concentrator. The resultant pellets were washed with $1 \mathrm{~mL}$ methanol, and lipid A was extracted using $100 \mu \mathrm{L}$ of chloroform, methanol, and water (3:1:0.25). After centrifugation at $2000 \times g$ for $15 \mathrm{~min}, 2 \mu \mathrm{L}$ of the supernatants were mixed with $2 \mu \mathrm{L}$ of $10 \mathrm{mg} / \mathrm{mL}$ norharmane matrix in chloroform:methanol (2:1), and $0.5 \mu \mathrm{L}$ of the mixtures were spotted on a matrix-assisted laser desorption-time of flight (MALDI-TOF) plate (5800 MALDI TOF/TOF Analyzer, Sciex, Ontario, Canada). Samples were analyzed in the negative-ion mode with reflectron mode. Calibration was performed using default calibration originated by five standard spots. Spectral data were analyzed with the 4000 Series Explorer software Version 4.1.0 (Sciex, Ontario, Canada), and used to estimate lipid A forms based on their predicted structures and molecular weights [10]. 


\subsection{Detergent Sensitivity Assay}

Sensitivity to the lytic effect of sodium dodecyl sulphate (SDS) was assessed by determining the turbidity $\left(\mathrm{OD}_{600}\right)$ of bacterial cells from late-exponential cultures resuspended in saline after incubation at room temperature for 5 minutes in the presence of increasing concentrations of SDS $(0-5 \%, w / v)$ [22].

\subsection{Antibiotics Sensitivity Assays}

Sensitivity to many different antibiotics was assessed by the Kirby-Bauer disc diffusion test. Bacterial cell suspensions in saline were normalized at $0.5 \mathrm{McFarland}$ Standard and swabbed onto MH agar plates, using disks containing ciprofloxacin $(5 \mu \mathrm{g})$, novobiocin $(30 \mu \mathrm{g})$, rifampicin $(5 \mu \mathrm{g})$, erythromycin $(15 \mu \mathrm{g})$, streptomycin $(10 \mu \mathrm{g})$, tobramycin $(10 \mu \mathrm{g})$, imipenem $(10 \mu \mathrm{g})$, and colistin $(10 \mu \mathrm{g})$ (Becton Dickinson). Growth inhibition halos were measured after $24 \mathrm{~h}$ of growth at 22,30 , or $37^{\circ} \mathrm{C}$.

Colistin sensitivity was also assessed with minimum inhibitory concentration (MIC) assay using the broth microdilution method. Briefly, strains were cultured in $\mathrm{MH}$ at $37^{\circ} \mathrm{C}$ for $8 \mathrm{~h}$, and then refreshed at $5 \times 10^{5}$ cells $/ \mathrm{ml}$ in the same medium in the presence of increasing concentrations of colistin (up to $16 \mu \mathrm{g} / \mathrm{mL}$ ). MIC was defined as the lowest concentration of antibiotics for which no visible growth was observed after $24 \mathrm{~h}$ at $37^{\circ} \mathrm{C}$. Each strain was tested in at least three independent experiments.

\subsection{Biofilm Assay}

Bacterial attachment and biofilm formation were evaluated through the microtiter plate biofilm assay. Bacteria were refreshed in $\mathrm{MH}$ from mid-exponential cultures at an $\mathrm{OD}_{600}=0.01$ and aliquoted in 96-well polystyrene plates ( $180 \mu \mathrm{L}$ per well). Plates were incubated for $24 \mathrm{~h}$ at 22,30 , or $37^{\circ} \mathrm{C}$ under static conditions. The wells were washed several times with distilled water, the attached cells were stained with $220 \mu \mathrm{L}$ of $0.1 \%$ crystal violet at room temperature for $15 \mathrm{~min}$, and the wells were washed again several times with distilled water to remove unbound dye. Biofilm-bound crystal violet was eluted with $200 \mu \mathrm{L}$ of $30 \%$ acetic acid at room temperature for $15 \mathrm{~min}$ and, for each well, $100 \mu \mathrm{L}$ of the resulting solution were aliquoted in a new microtiter plate. The released crystal violet was measured as $\mathrm{OD}_{595}$ in a Tecan Spark $10 \mathrm{M}$ microtiter reader. Four biological replicates with four wells each were performed for each strain.

\subsection{Whole Blood Killing Assay}

Whole blood killing assay was performed as described [13], with few modifications. Briefly, bacteria were grown in $\mathrm{MH}$ until the late-exponential phase, harvested by centrifugation and resuspended in saline at $\mathrm{OD}_{600}=1$. Three hundred microliters of fresh human blood, pooled from three healthy donors, were mixed with $100 \mu \mathrm{L}$ of bacterial suspensions, and incubated at $37^{\circ} \mathrm{C}$ and $220 \mathrm{rpm}$. Serial dilutions in saline were plated onto $\mathrm{MH}$ agar plate at time 0 and after 1-h incubation with human blood to obtain viable counts. Human serum was obtained from healthy volunteers who gave their written informed consent to the study. The research project was approved by the review board of the Pasteur Institute-Cenci Bolognetti Foundation, Sapienza University of Rome (Rome, Italy).

\subsection{Galleria Mellonella Infection and Persistence Assays}

G. mellonella larvae were purchased from the Serpens breeding (Paliano, Italy; www.bigserpens.com) and used within one day of shipment. P. aeruginosa strains were grown in MH until mid-exponential phase of growth, and serial ten- or three-fold dilutions of bacterial cell suspensions in saline were injected into G. mellonella larvae as described [23]. Infected larvae were incubated at $30^{\circ} \mathrm{C}$ for up to 3 days to monitor mortality. Ten larvae were infected with each infecting dose in three independent assays. Kaplan-Meier survival curves, lethal doses $90 \%\left(\mathrm{LD}_{90}\right)$, and $\mathrm{R}^{2}$ values were obtained using GraphPad Prism as described [24].

P. aeruginosa persistence in G. mellonella larvae was assessed as described [22], with few modifications. Briefly, larvae were infected with about $10^{6}$ bacterial cells and, after 2,4 , or $8 \mathrm{~h}$ 
of incubation at $30{ }^{\circ} \mathrm{C}$, cut with a razor blade to recover the hemolymph. Ten-fold serial dilutions of the hemolymph were plated on MH agar supplemented with $100 \mu \mathrm{g} / \mathrm{mL}$ ampicillin (to which P. aeruginosa is intrinsically resistant) to determine the percentage of viable cells with respect to the infecting dose.

P. aeruginosa persistence in G. mellonella hemolymph was also assessed ex vivo. About $10^{6}$ bacterial cells were inoculated in the hemolymph collected and pooled from ten larvae and, after 2, 4, or $8 \mathrm{~h}$ of incubation at $30^{\circ} \mathrm{C}$, the samples were serially diluted in saline and plated on $\mathrm{MH}$ agar supplemented with $100 \mu \mathrm{g} / \mathrm{mL}$ ampicillin.

\subsection{Statistical Analyses}

Statistical analysis was performed with GraphPad Instat, using One-Way Analysis of Variance (ANOVA) followed by Tukey-Kramer multiple comparison test. Statistical analysis of survival curves of G. mellonella larvae or bacterial persistence in larvae was performed with GraphPad Prism, using the Log-rank (Mantel-Cox) test or the Kruskal-Wallis test followed by Dunn's multiple comparison test, respectively.

\section{Results and Discussion}

\subsection{Both LpxO1 and LpxO2 are Responsible for Lipid A Hydroxylation}

In order to verify the involvement of $l p x O$ homologs in the hydroxylation of secondary $\mathrm{C} 12$ chains of lipid A in P. aeruginosa, single and double in-frame deletion mutants in lpxO1 (PA4512) and/or $1 p x O 2$ (PA0936) were generated in the reference laboratory strain PAO1. Lipid A was extracted from mutant and wild type cells and analyzed by MALDI-TOF mass spectrometry. In line with previous evidence [10], the main lipid A form of the wild type strain PAO1 is the diphosphorylated penta-acylated lipid A, with a fraction of this molecule modified by the addition of palmitoyl group to the $\mathrm{C} 10$ acyl chain (Figure 2A). For both these lipid A variants, the MALDI spectra showed three distinct peaks with $\mathrm{m} / \mathrm{z}$ differences of 16 , due to the presence/absence of a hydroxyl group in the secondary C12 acyl chains [4], although the peaks corresponding to the monohydroxylated forms $(\mathrm{m} / \mathrm{z}=1446$ and 1684) were much more abundant (Figure 2A). Notably, a reduction in the lipid A hydroxylation state was observed in all $l p x O$ mutants. In particular, the $\Delta l p x O 1$ mutant lost the peaks corresponding to dihydroxylated lipid $\mathrm{A}$, but the monohydroxylated forms remained predominant. In contrast, the lipid A of the $\Delta l p x O 2$ mutant was mainly present in the non-hydroxylated state $(\mathrm{m} / \mathrm{z}=1430$ and 1668$)$, although monohydroxylated peaks were also detected. Finally, only non-hydroxylated lipid A was observed in the $\Delta l p x O 1 \Delta l p x O 2$ double mutant (Figure 2A). Besides the hydroxylation state, a slight increase was observed in the relative intensity of peaks corresponding to tetra-acylated lipid $\mathrm{A}$ in all mutants and of triphosphorylated lipid A forms in $\triangle l p x O 2$ strains only (Figure 2A). While these lipid A forms were previously observed in P. aeruginosa [25-27], whether they have any effects on the properties of the LPS layer is not known. Ectopic expression of lpxO2 in the $\triangle l p x \mathrm{O} 2$ mutant from an IPTG-inducible promoter restored the lipid A hydroxylation pattern of the wild type (Figure S1). In contrast, IPTG-induced expression of $l p x \mathrm{O} 1$ in the $\Delta l p x O 1$ mutant significantly increased the relative intensity of dihydroxylated lipid A peaks (Figure S1), suggesting that the minor effect of lpxO1 deletion on lipid A hydroxylation (Figure 2A) is likely due to poor expression and/or activity of the endogenous lpxO1 gene under the growth conditions tested in this work. 
A
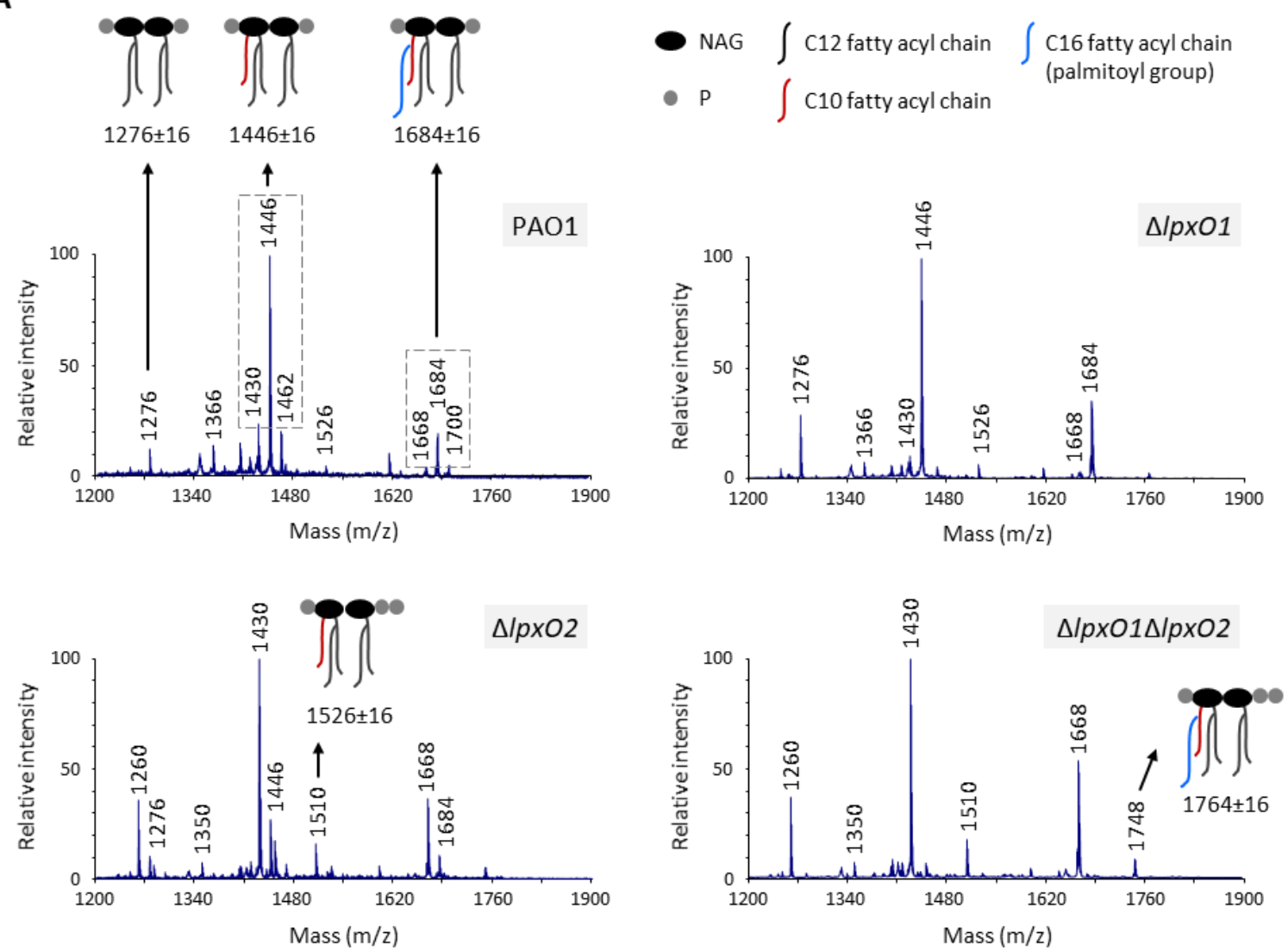

B
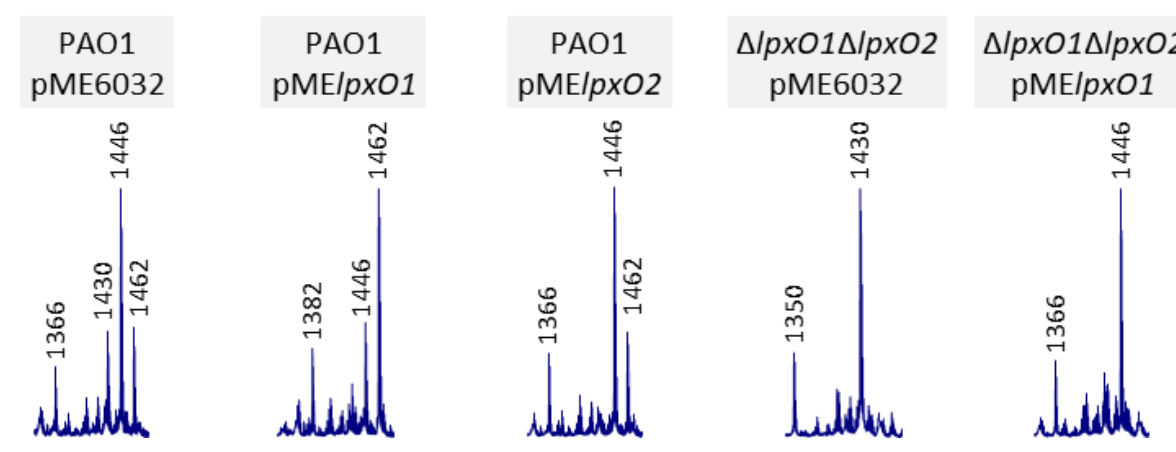

$\triangle / p \times 01 \Delta / p \times O 2$ pMEIpxO2

Figure 2. Role of LpxO1 and LpxO2 in lipid A hydroxylation. (A) MALDI-TOF analysis of lipid A extracted from $P$. aeruginosa wild type (PAO1) and lpxO1 and/or lpxO2 mutant cells cultured in Mueller-Hinton broth $(\mathrm{MH})$ at $37^{\circ} \mathrm{C}$ until the late-exponential growth phase. Spectra were obtained in the ion negative mode, thus $\mathrm{m} / \mathrm{z}$ values correspond to (molecular mass -1 )/ 1 , and are representative of three biological replicates. Relevant lipid A forms are shown with cartoons: $\mathrm{m} / \mathrm{z}=1276$, diphosphorylated tetra-acylated lipid A; $\mathrm{m} / \mathrm{z}=1446$, diphosphorylated penta-acylated lipid A; m/z = 1526, triphosphorylated penta-acylated lipid A; $\mathrm{m} / \mathrm{z}=1684$, diphosphorylated penta-acylated lipid A with a palmitoyl group; and $\mathrm{m} / \mathrm{z}=1764$, triphosphorylated penta-acylated lipid A with a palmitoyl group. $\mathrm{m} / \mathrm{z} \pm 16$ indicates lipid $\mathrm{A}$ forms that vary for the presence/absence of a hydroxyl group to each secondary C12 acyl chain. (B) Details of the MALDI-TOF spectra of lipid A extracted from PAO1 or the $\Delta l p x \mathrm{O} 1 \Delta l p x \mathrm{O} 2$ mutant carrying constructs for LpxO1 or LpxO2 overexpression (pMElpxO1 or pMElpxO2) or the empty vector pME6032, cultured in the presence of $0.5 \mathrm{mM}$ isopropyl-1-thio-ß-D-galactopyranoside (IPTG). The images highlight the diphosphorylated penta-acylated lipid A species at $\mathrm{m} / \mathrm{z}=1430,1446$, and 1462 (non-, mono-, and dihydroxylated, respectively), while the corresponding full-length spectra are shown in Figure S2. 
Altogether, these data demonstrate that both LpxO homologs of P. aeruginosa can hydroxylate the secondary $\mathrm{C} 12$ chains of lipid A, although LpxO2 appears to be responsible for most of lipid A hydroxylation, at least in the reference strain PAO1 and/or in our experimental setting. Moreover, the complete lack of dihydroxylated lipid A forms in both lpxO1 and lpxO2 single mutants, which instead retain mono-hydroxylated species (Figure 2A), strongly suggests that each LpxO enzyme hydroxylates a specific $\mathrm{C} 12$ chain. This hypothesis was confirmed by assessing the effect of individual LpxO1 or LpxO2 overexpression in wild type and $\Delta l p x O 1 \Delta l p x O 2$ backgrounds. In the wild type strain, $\mathrm{LpxO} 1$ overexpression strongly increased the relative intensity of dihydroxylated lipid A peaks, while $\mathrm{LpxO} 2$ overexpression only led to the loss of non-hydroxylated forms (Figure 2B and Figure S2). This confirms that, in our experimental setting, LpxO2 is the most active enzyme in PAO1, while LpxO1 is likely poorly expressed from its endogenous promoter. On the other hand, overexpression of either LpxO1 or LpxO2 in the $\Delta l p x \mathrm{O} 1 \Delta l p x \mathrm{O} 2$ double mutant restored monohydroxylation of lipid A, but dihydroxylated species were not observed for any overexpressed enzyme (Figure 2B and Figure S2). This finding further supports the hypothesis that the two LpxO proteins of P. aeruginosa have specificity for different $\mathrm{C} 12$ chains. Unfortunately, our attempts to distinguish the position of the hydroxyl groups by MALDI-TOF/TOF tandem mass spectrometry analysis of monohydroxylated lipid A extracted from the $\Delta l p x O 1 \Delta l p x O 2$ mutant individually overexpressing each LpxO homolog did not clarify this issue (data not shown). Further experiments and/or different genetics and biochemical approaches will therefore be required to elucidate the specificity of the two P. aeruginosa LpxO enzymes.

\subsection{Lipid A Hydroxylation Does Not Affect in vitro Growth, Cell Envelope Integrity and Resistance}

Since the parental strain and the different $l p x O$ mutants showed various degrees of lipid $\mathrm{A}$ hydroxylation (Figure 2), we used these strains to assess whether lipid A hydroxylation can affect $P$. aeruginosa physiology. First, we compared planktonic growth among the wild type and lpxO mutant strains. Growth kinetics and yields were almost identical, both in complex (Figure 3A) and minimal media (Figure S3). In addition, only minor, non-significant differences were observed in biofilm formation between wild type and lpxO mutants (Figure 3B), indicating that lipid A hydroxylation is not required for both planktonic and biofilm growth in P. aeruginosa.

We then assessed the role of lipid A hydroxylation in antibiotic resistance, by comparing the activity of different antibiotics with different chemical properties and mechanisms of action against the wild type and $l p x O$ mutant strains through the Kirby-Bauer disc diffusion assay. As reported in Table 1, we did not observe any variation in drug resistance in any mutant and for any antibiotic. Since the poor agar diffusion of colistin hampers the detection of minor differences in resistance through the disk diffusion test [28], and considering that lipid A hydroxylation was found to affect polymyxin resistance in many different bacteria, such as Vibrio cholerae, K. pneumonia, and A. baumannii [13,14,29], we confirmed that all strains have the same colistin MIC using the broth microdilution method (Table 1). These results rule out any specific effect of the hydroxylation state of lipid A on antibiotic resistance, as well as any general role in the permeability barrier of the P. aeruginosa outer membrane. This is in line with the finding that all strains showed a comparable resistance profile to the lytic effect of the detergent SDS (Figure 3C), which has been previously used to reveal defects in outer membrane stability $[7,10,22]$, thus implying that the integrity of the cell envelope of P. aeruginosa PAO1 is not affected by changes in the lipid A hydroxylation levels. 
A

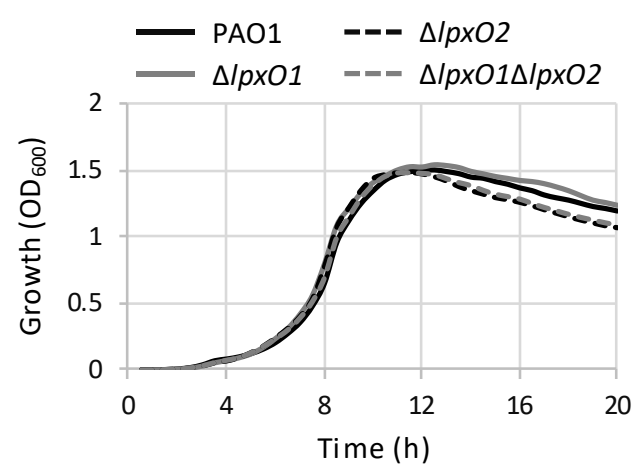

C

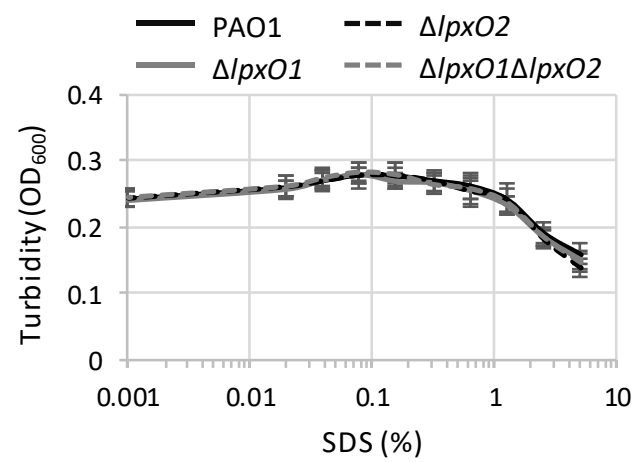

B

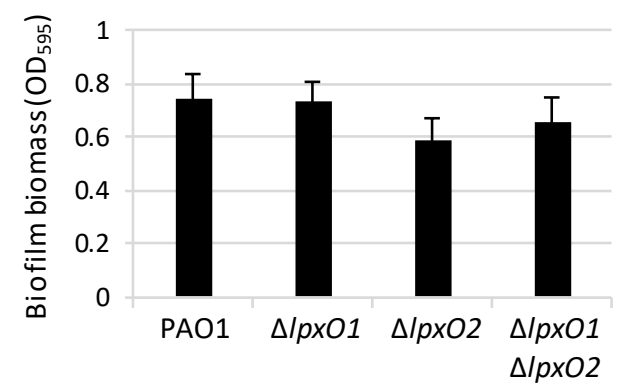

D

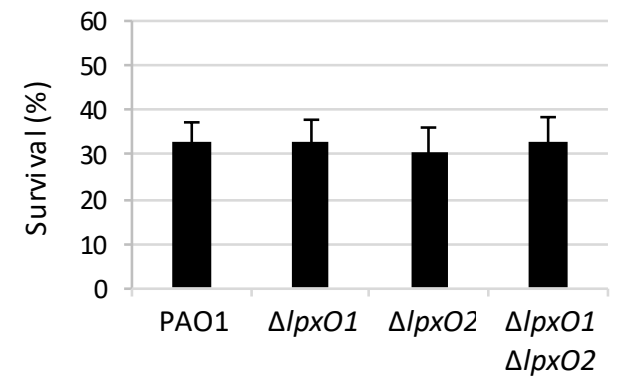

Figure 3. Effect of lipid A hydroxylation on P. aeruginosa planktonic and biofilm growth cell envelope integrity and human blood resistance. (A) Planktonic growth $\left(\mathrm{OD}_{600}\right)$ of the wild type PAO1 and $\Delta l p x \mathrm{O} 1, \Delta l p x \mathrm{O} 2$, and $\Delta l p x \mathrm{O} 1 \Delta l p x \mathrm{O} 2$ deletion mutants in $\mathrm{MH}$ at $37^{\circ} \mathrm{C}$. Growth curves are representative of three independent experiments performed in triplicate. (B) Biofilm formation in 96-well polystyrene microtiter plates of PAO1 and $l p x O$ mutants after $24 \mathrm{~h}$ at $37^{\circ} \mathrm{C}$ under static conditions. Values are the mean $( \pm$ SD) from four independent assays. (C) Lytic effect of sodium dodecyl sulphate (SDS) at different concentrations $(0 \%-5 \%)$, measured as a decrease in cell suspension turbidity $\left(\mathrm{OD}_{600}\right)$, on wild type and $l p x O$ mutant cells. Values are the mean $( \pm \mathrm{SD})$ from three independent experiments performed in duplicate. (D) Survival of PAO1 and lpxO mutant cells in whole human blood after 1-h incubation at $37^{\circ} \mathrm{C}$. Values are the mean $( \pm \mathrm{SD})$ from three independent experiments, and are expressed as percentage of viable cells with respect to the initial inoculum. Differences were not statistically significant $(P>0.05$; ANOVA).

Table 1. Antibiotic susceptibility of P. aeruginosa lp $x O$ mutants by the Kirby-Bauer disk diffusion and/or minimum inhibitory concentration (MIC) assays ${ }^{1}$.

\begin{tabular}{|c|c|c|c|c|c|c|c|c|c|}
\hline \multirow{2}{*}{ Strain } & \multicolumn{8}{|c|}{ Inhibition Halo $(\mathrm{mm})^{2}$} & \multirow{2}{*}{$\begin{array}{c}\begin{array}{c}\text { MIC } \\
(\mu \mathrm{g} / \mathrm{ml})\end{array} \\
\mathrm{Ct}\end{array}$} \\
\hline & Cip & Novo & Rif & Ery & Sm & Tob & $\operatorname{Imp}$ & $\mathrm{Ct}$ & \\
\hline$\Delta l p x O 1$ & $30.8( \pm 1.6)$ & $6.0( \pm 0.0)$ & $6.0( \pm 0.0)$ & $6.0( \pm 0.0)$ & $12.6( \pm 0.7)$ & $23.2( \pm 3.1)$ & $24.2( \pm 0.5)$ & $12.5( \pm 0.5)$ & 0.5 \\
\hline$\Delta l p x \mathrm{O} 2$ & $30.9( \pm 1.1)$ & $6.0( \pm 0.0)$ & $6.0( \pm 0.0)$ & $6.0( \pm 0.0)$ & $11.9( \pm 1.5)$ & $22.0( \pm 0.9)$ & $25.2( \pm 0.8)$ & $12.5( \pm 0.5)$ & 0.5 \\
\hline$\Delta l p x O 1 \Delta l p x O 2$ & $30.8( \pm 2.2)$ & $6.0( \pm 0.0)$ & $6.0( \pm 0.0)$ & $6.0( \pm 0.0)$ & $12.4( \pm 1.8)$ & $23.0( \pm 2.8)$ & $24.4( \pm 1.2)$ & $12.5( \pm 0.5)$ & 0.5 \\
\hline
\end{tabular}

${ }^{1}$ Abbreviations: Cip, ciprofloxacin; Novo, novobiocin; Rif, rifampicin; Ery, erythromycin; Sm, streptomycin; Tob, tobramycin; Ipm, imipenem; Ct, colistin. ${ }^{2}$ Values are the mean ( $\left.\pm \mathrm{SD}\right)$ of four independent assays. ${ }^{3}$ Values are the mode of six independent assays.

Whole blood killing assay has been recently used to highlight the contribution of lipid A hydroxylation to resistance to professional phagocytes in A. baumannii and K. pneumoniae [13,14]. We therefore sought to verify ex vivo whether LpxO1 and/or LpxO2 affect P. aeruginosa PAO1 survival in human blood. Once again, none of the $l p x O$ mutants showed significant differences in survival in 
whole human blood with respect to their parental strain (Figure 3D), indicating that hydroxylation in the lipid A secondary chains does not contribute to blood resistance in P. aeruginosa.

Notably, lpxO1 was found to be slightly up-regulated (about two-fold) at $25^{\circ} \mathrm{C}$ as compared to $37^{\circ} \mathrm{C}$ by transcriptomic analysis [30], and a possible correlation between a specific single nucleotide polymorphism (SNP) in $l p x O 1$ and biofilm formation at $22{ }^{\circ} \mathrm{C}$ has been recently proposed through genome-wide association analysis [31]. To investigate any possible temperature-dependent effects of LpxO enzymes, we performed growth, biofilm, and antibiotic resistance assays also at 22 and $30{ }^{\circ} \mathrm{C}$. As shown in Figure S4, we did not observe any relevant differences between the wild type and lpxO mutants under both conditions. While this further confirms that lipid A hydroxylation is not required for growth, biofilm formation, and for proper permeability barrier of the outer membrane, we cannot rule out that it may have more subtle effects on P. aeruginosa physiology that cannot be highlighted by our assays.

\subsection{Lipid A Hydroxylation Contributes to P. aeruginosa Virulence in an Insect Model of Infection}

Finally, to gain insights into the possible role of lipid A hydroxylation during P. aeruginosa PAO1 infection, we assessed the pathogenicity of lipid A hydroxylation-defective mutants in the well-established G. mellonella model of infection [32], which represents a convenient and easy-to-handle infection model to preliminary screen the infectivity of P. aeruginosa mutants [23].

Differently from all the in vitro results, mutants impaired in lipid A hydroxylation were found to be significantly attenuated in pathogenicity in G. mellonella larvae. Indeed, we observed a fourand almost eight-fold increase in the $\mathrm{LD}_{90}$ of the $\Delta l p x \mathrm{O} 2$ and $\Delta l p x \mathrm{O} 1 \Delta l p x \mathrm{O} 2$ mutants with respect to the wild type, respectively (Figure $4 \mathrm{~A}$ ). The reduced infectivity of these two mutants was confirmed by Kaplan-Maier survival curves obtained with a definite infecting dose (about 15 bacterial cells) (Figure $4 \mathrm{~B}$ ). In contrast, both the $\mathrm{LD}_{90}$ values and the timing of killing of the $\Delta l p x O 1$ mutant were basically identical to those of the parental strain (Figures $4 \mathrm{~A}$ and $4 \mathrm{~B}$ ), indirectly implying that LpxO2 is the main enzyme involved in lipid A hydroxylation during G. mellonella infection, in agreement with in vitro observations (Figure 2).

In order to assess whether the reduced infectivity of mutants defective in lipid A hydroxylation could be due to impaired persistence of bacterial cells in vivo during the infection, G. mellonella larvae were infected with a high infecting dose (about $10^{6} \mathrm{CFUs}$ ) and the number of $P$. aeruginosa cells in the hemolymph was determined at 2,4 , and $8 \mathrm{~h}$ post-infection. We only observed a slight delay in growth in G. mellonella larvae for the $\Delta l p x O 2$ mutant and the $\triangle l p x O 1 \Delta l p x O 2$ double mutant, although the difference with the wild type was statistically significant only for $\Delta l p x \mathrm{O} 1 \Delta l p x \mathrm{O} 2$ at $4 \mathrm{~h}$ (Figure $4 \mathrm{C}$ ). Notably, no significant differences between wild type and any lp $x O$ mutant were observed when bacterial cell viability was assessed ex vivo in the hemolymph extracted from G. mellonella larvae (Figure S5). This result, together with those obtained from in vitro assays, suggests that LpxO enzyme(s) and lipid A hydroxylation could contribute to P. aeruginosa in vivo growth and virulence in G. mellonella larvae in a manner that does not directly depend on the integrity of the cell envelope and/or resistance to the antimicrobial peptides of the G. mellonella hemolymph. 
A
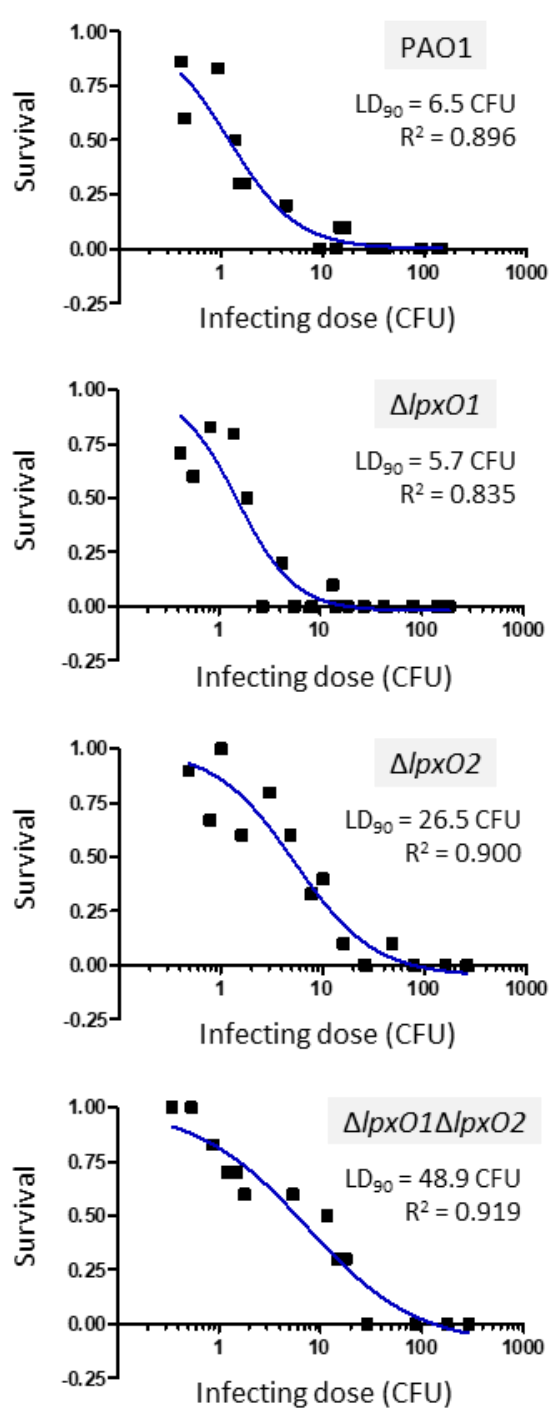

B

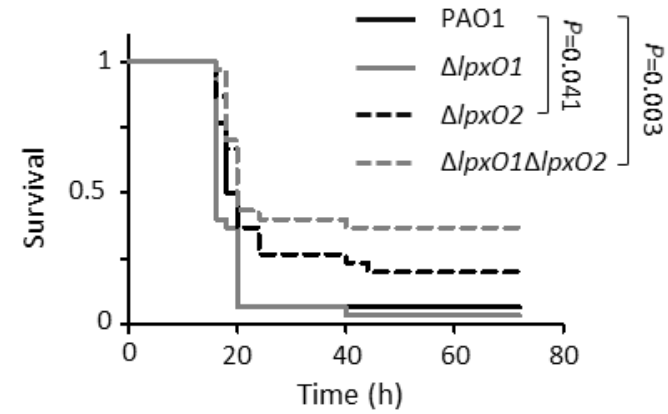

C

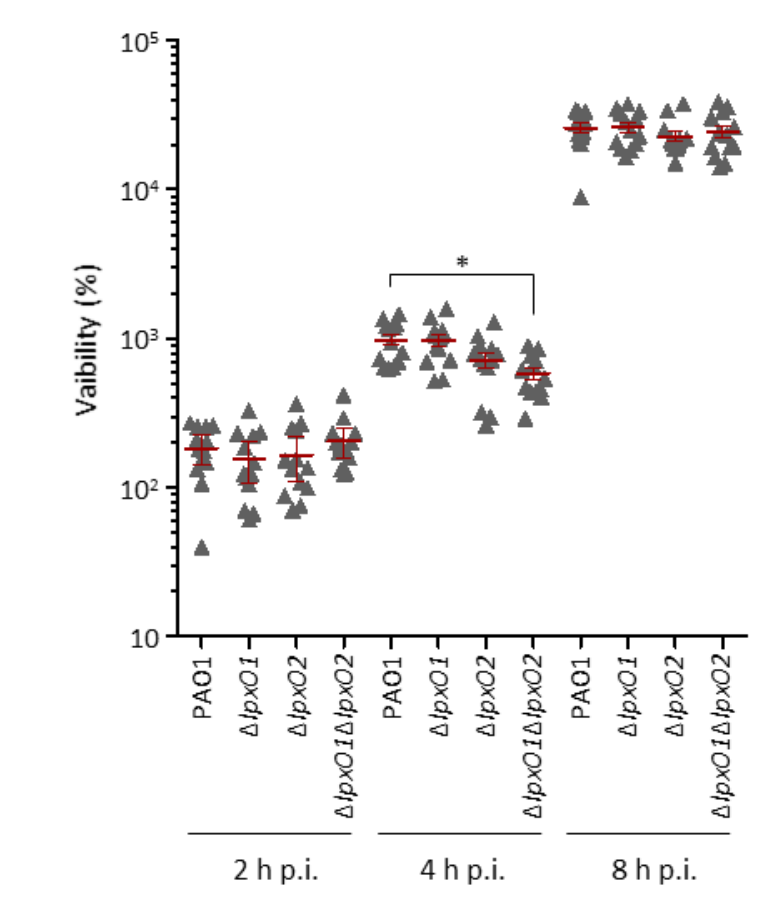

Figure 4. Effect of lipid A hydroxylation on P. aeruginosa infectivity in G. mellonella. (A) Dose-dependent survival curves of G. mellonella larvae infected with different doses of the wild type PAO1, the $\Delta l p x O 1$, the $\Delta l p x O 2$, or the $\Delta l p x O 1 \Delta l p x O 2$ deletion mutant. Ten larvae were infected with each infecting dose in three independent experiments. $\mathrm{LD}_{90}$ and $\mathrm{R}^{2}$ values were determined by GraphPad Prism and are reported in the figure. Abbreviation: CFU, colony-forming units. (B) Kaplan-Meier survival curves of G. mellonella larvae infected with PAO1 (14.9 $\pm 1.6 \mathrm{CFU}), \Delta l p x \mathrm{O} 1$ (15.4 $\pm 2.9 \mathrm{CFU}), \Delta l p x \mathrm{O} 2$ $(14.0 \pm 3.4 \mathrm{CFU})$, or $\Delta l p x O 1 \Delta l p x O 2(14.8 \pm 3.2 \mathrm{CFU})$. Thirty larvae were infected with each strain in three independent experiments. Only statistically significant differences are shown in the figure, according to the Log-rank (Mantel-Cox) test. (C) Viability of PAO1, $\Delta l p x O 1, \Delta l p x O 2$, or $\Delta l p x O 1 \Delta l p x O 2$ cells in G. mellonella larvae at 2, 4, and $8 \mathrm{~h}$ post-infection (p.i.), reported as a percentage with respect to the infecting dose. Means with $95 \%$ confidence intervals are shown in red. For each time point, thirteen larvae per strain were infected in three independent assays. The asterisk indicates a statistically significant difference $(P<0.05)$ with respect to the wild type at the same time point (Kruskal-Wallis).

\section{Conclusions}

Several studies on different Gram-negative pathogens have highlighted the importance of lipid A modifications in the adaptation of the outer membrane to harsh environments and to the host during infections [33-36]. In P. aeruginosa, the enzymes responsible for the addition/removal of phosphate, palmitoyl, hydroxydecanoyl, and lauryl groups to/from lipid A, as well as their effects on 
outer membrane remodeling and bacterial physiology, have been characterized [9,37-39]. In contrast, the role of hydroxylation of secondary lipid A fatty acid chains was not previously examined in this species, although P. aeruginosa is the only Gram-negative pathogen reported so far with two hydroxylated secondary fatty acids in lipid A. Here, we demonstrated that the two lpxO homologs of P. aeruginosa are both functional and hydroxylate different secondary chains, even if $l p x O 2$ accounts for the majority of lipid A hydroxylation, at least in the reference strain PAO1 and under the in vitro and in vivo conditions investigated in our study. Interestingly, we observed that, differently from other bacterial pathogens, lipid A hydroxylation does not affect the integrity and permeability barrier of the P. aeruginosa outer membrane, and accordingly is not required for in vitro growth, ex vivo blood resistance, and persistence in G. mellonella hemolymph. Nevertheless, we found that mutants impaired in lipid A hydroxylation are significantly attenuated in virulence in the G. mellonella model of acute infection. This suggests that lipid A hydroxylation could also play some role in bacterial virulence other than (or additional to) its previously reported effect on outer membrane integrity and resistance. This work paves the way for future studies aimed at confirming the importance of lipid A hydroxylation in other P. aeruginosa infection models, and/or at unravelling the molecular basis of lipid A hydroxylation-dependent effect on P. aeruginosa virulence.

Supplementary Materials: The following are available online at http://www.mdpi.com/2076-0817/8/4/291/s1, Figure S1: MALDI-TOF lipid A spectra of $\triangle l p x \mathrm{O} 1$ and $\Delta l p x \mathrm{O} 2$ mutants carrying the complementing plasmid pMElpxO1 or pMElpxO2, Figure S2: MALDI-TOF lipid A spectra of the PAO1 and the $\triangle l p x O 1 \Delta l p x O 2$ mutant overexpressing LpxO1 or LpxO2, Figure S3: Bacterial growth curves in minimal medium, Figure S4: Growth, biofilm formation and antibiotic sensitivity profile at 22 and $30^{\circ} \mathrm{C}$, Figure S5: Bacterial viability in the G. mellonella hemolymph ex vivo, Table S1: Bacterial strains and plasmids used in this study, Table S2: Primers used in this study.

Author Contributions: Conceptualization, A.L.S. and F.I.; formal analysis, F.I.; investigation, A.L.S., M.C., R.S., M.C.S. and A.D.G.; data curation, A.L.S., M.C., C.M. and F.I.; writing-original draft preparation, F.I.; writing - review and editing, A.L.S., M.C., R.S., M.C.S., A.D.G., C.M. and F.I.; supervision, C.M. and F.I.; project administration, F.I.; funding acquisition, F.I.

Funding: This work was supported by the Excellence Departments grant from the Italian Ministry of Education, University and Research (MIUR, Italy) (Art. 1, commi 314-337 Legge 232/2016) to the Department of Science, Roma Tre University, by the Pasteur Institute-Cenci Bolognetti Foundation and by the PRIN 2017 grant protocol 20177J5Y3P.

Acknowledgments: We are grateful to Prof. Robert Ernst for sharing the protocol and suggestions for the extraction and analysis of lipid A, and to the personnel of Policlinico Umberto I (Sapienza University of Rome) for their help in collecting blood samples from healthy donors.

Conflicts of Interest: The authors declare no conflict of interest.

\section{References}

1. Silhavy, T.J.; Kahne, D.; Walker, S. The bacterial cell envelope. Cold Spring Harb. Perspect. Biol. 2010, 2, a000414. [CrossRef] [PubMed]

2. Nikaido, H. Molecular basis of bacterial outer membrane permeability revisited. Microbiol. Mol. Biol. Rev. 2003, 67, 593-656. [CrossRef] [PubMed]

3. Tümmler, B. Emerging therapies against infections with Pseudomonas aeruginosa. F1000Research 2019, 8, F1000. [CrossRef] [PubMed]

4. King, J.D.; Kocíncová, D.; Westman, E.L.; Lam, J.S. Review: Lipopolysaccharide biosynthesis in Pseudomonas aeruginosa. Innate Immun. 2009, 15, 261-312. [CrossRef]

5. Mdluli, K.E.; Witte, P.R.; Kline, T.; Barb, A.W.; Erwin, A.L.; Mansfield, B.E.; McClerren, A.L.; Pirrung, M.C.; Tumey, L.N.; Warrener, P.; et al. Molecular validation of LpxC as an antibacterial drug target in Pseudomonas aeruginosa. Antimicrob. Agents Chemother. 2006, 50, 2178-2184. [CrossRef]

6. Srinivas, N.; Jetter, P.; Ueberbacher, B.J.; Werneburg, M.; Zerbe, K.; Steinmann, J.; Van der Meijden, B.; Bernardini, F.; Lederer, A.; Dias, R.L.; et al. Peptidomimetic antibiotics target outer-membrane biogenesis in Pseudomonas aeruginosa. Science 2010, 327, 1010-1013. [CrossRef]

7. Fernández-Piñar, R.; Lo Sciuto, A.; Rossi, A.; Ranucci, S.; Bragonzi, A.; Imperi, F. In vitro and in vivo screening for novel essential cell-envelope proteins in Pseudomonas aeruginosa. Sci. Rep. 2015, 5, 17593. [CrossRef] 
8. Knirel, Y.A.; Bystrova, O.V.; Kocharova, N.A.; Zähringer, U.; Pier, G.B. Conserved and variable structural features in the lipopolysaccharide of Pseudomonas aeruginosa. J. Endotoxin Res. 2006, 12, 324-336. [CrossRef]

9. $\quad$ Ernst, R.K.; Adams, K.N.; Moskowitz, S.M.; Kraig, G.M.; Kawasaki, K.; Stead, C.M.; Trent, M.S.; Miller, S.I. The Pseudomonas aeruginosa lipid A deacylase: Selection for expression and loss within the cystic fibrosis airway. J. Bacteriol. 2006, 188, 191-201. [CrossRef]

10. Lo Sciuto, A.; Martorana, A.M.; Fernández-Piñar, R.; Mancone, C.; Polissi, A.; Imperi, F. Pseudomonas aeruginosa LptE is crucial for LptD assembly, cell envelope integrity, antibiotic resistance and virulence. Virulence 2018, 9, 1718-1733. [CrossRef]

11. Gibbons, H.S.; Lin, S.; Cotter, R.J.; Raetz, C.R. Oxygen requirement for the biosynthesis of the S-2-hydroxymyristate moiety in Salmonella typhimurium lipid A. Function of LpxO, A new Fe ${ }^{2+} / \mathrm{alpha}^{-}$ ketoglutarate-dependent dioxygenase homologue. J. Biol. Chem. 2000, 275, 32940-32949. [CrossRef] [PubMed]

12. Gibbons, H.S.; Reynolds, C.M.; Guan, Z.; Raetz, C.R. An inner membrane dioxygenase that generates the 2-hydroxymyristate moiety of Salmonella lipid A. Biochemistry 2008, 47, 2814-2825. [CrossRef] [PubMed]

13. Mills, G.; Dumigan, A.; Kidd, T.; Hobley, L.; Bengoechea, J.A. Identification and Characterization of Two Klebsiella pneumoniae lpxL Lipid A Late Acyltransferases and Their Role in Virulence. Infect. Immun. 2017, 85, e00068-17. [CrossRef] [PubMed]

14. Bartholomew, T.L.; Kidd, T.J.; Sá Pessoa, J.; Conde Álvarez, R.; Bengoechea, J.A. 2-Hydroxylation of Acinetobacter baumannii Lipid A Contributes to Virulence. Infect. Immun. 2019, 87, e00066-19. [CrossRef] [PubMed]

15. Norris, M.H.; Somprasong, N.; Schweizer, H.P.; Tuanyok, A. Lipid A Remodeling Is a Pathoadaptive Mechanism That Impacts Lipopolysaccharide Recognition and Intracellular Survival of Burkholderia pseudomallei. Infect. Immun. 2018, 86, e00360-18. [CrossRef] [PubMed]

16. Llobet, E.; Martínez-Moliner, V.; Moranta, D.; Dahlström, K.M.; Regueiro, V.; Tomás, A.; Cano, V.; Pérez-Gutiérrez, C.; Frank, C.G.; Fernández-Carrasco, H.; et al. Deciphering tissue-induced Klebsiella pneumoniae lipid A structure. Proc. Natl. Acad. Sci. USA 2015, 112, E6369-E6378. [CrossRef] [PubMed]

17. Rocchio, S.; Santorelli, D.; Rinaldo, S.; Franceschini, M.; Malatesta, F.; Imperi, F.; Federici, L.; Travaglini-Allocatelli, C.; Di Matteo, A. Structural and functional investigation of the Small Ribosomal Subunit Biogenesis GTPase A (RsgA) from Pseudomonas aeruginosa. FEBS J. 2019. [CrossRef]

18. Milton, D.L.; O'Toole, R.; Horstedt, P.; Wolf-Watz, H. Flagellin A is essential for the virulence of Vibrio anguillarum. J. Bacteriol. 1996, 178, 1310-1319. [CrossRef]

19. Hoang, T.T.; Karkhoff-Schweizer, R.R.; Kutchma, A.J.; Schweizer, H.P. A broad-host-range Flp-FRT recombination system for site-specific excision of chromosomally-located DNA sequences: Application for isolation of unmarked Pseudomonas aeruginosa mutants. Gene 1998, 212, 77-86. [CrossRef]

20. Heeb, S.; Blumer, C.; Haas, D. Regulatory RNA as mediator in GacA/RsmA-dependent global control of exoproduct formation in Pseudomonas fluorescens CHA0. J Bacteriol. 2002, 184, 1046-1056. [CrossRef]

21. Liu, Y.Y.; Chandler, C.E.; Leung, L.M.; McElheny, C.L.; Mettus, R.T.; Shanks, R.M.Q.; Liu, J.H.; Goodlett, D.R.; Ernst, R.K.; Doi, Y. Structural Modification of Lipopolysaccharide Conferred by mcr-1 in Gram-Negative ESKAPE Pathogens. Antimicrob. Agents Chemother. 2017, 61, e00580-17. [CrossRef] [PubMed]

22. Lo Sciuto, A.; Fernández-Piñar, R.; Bertuccini, L.; Iosi, F.; Superti, F.; Imperi, F. The periplasmic protein TolB as a potential drug target in Pseudomonas aeruginosa. PLoS ONE 2014, 9, e103784. [CrossRef] [PubMed]

23. Jander, G.; Rahme, L.G.; Ausubel, F.M. Positive correlation between virulence of Pseudomonas aeruginosa mutants in mice and insects. J. Bacteriol. 2000, 182, 3843-3845. [CrossRef] [PubMed]

24. Antunes, L.C.; Imperi, F.; Carattoli, A.; Visca, P. Deciphering the multifactorial nature of Acinetobacter baumannii pathogenicity. PLoS ONE 2011, 6, e22674. [CrossRef] [PubMed]

25. Cigana, C.; Curcurù, L.; Leone, M.R.; Ieranò, T.; Lorè, N.I.; Bianconi, I.; Silipo, A.; Cozzolino, F.; Lanzetta, R.; Molinaro, A.; et al. Pseudomonas aeruginosa exploits lipid A and muropeptides modification as a strategy to lower innate immunity during cystic fibrosis lung infection. PLoS ONE 2009, 4, e8439. [CrossRef]

26. Korneev, K.V.; Arbatsky, N.P.; Molinaro, A.; Palmigiano, A.; Shaikhutdinova, R.Z.; Shneider, M.M.; Pier, G.B.; Kondakova, A.N.; Sviriaeva, E.N.; Sturiale, L.; et al. Structural Relationship of the Lipid A Acyl Groups to Activation of Murine Toll-Like Receptor 4 by Lipopolysaccharides from Pathogenic Strains of Burkholderia mallei, Acinetobacter baumannii, and Pseudomonas aeruginosa. Front. Immunol. 2015, 6, 595. [CrossRef] 
27. Latino, L.; Caroff, M.; Pourcel, C. Fine structure analysis of lipopolysaccharides in bacteriophage-resistant Pseudomonas aeruginosa PAO1 mutants. Microbiology 2017, 163, 848-855. [CrossRef]

28. Galani, I.; Kontopidou, F.; Souli, M.; Rekatsina, P.D.; Koratzanis, E.; Deliolanis, J.; Giamarellou, H. Colistin susceptibility testing by Etest and disk diffusion methods. Int. J. Antimicrob. Agents 2008, 31, 434-439. [CrossRef]

29. Hankins, J.V.; Madsen, J.A.; Giles, D.K.; Childers, B.M.; Klose, K.E.; Brodbelt, J.S.; Trent, M.S. Elucidation of a novel Vibrio cholerae lipid A secondary hydroxy-acyltransferase and its role in innate immune recognition. Mol. Microbiol. 2011, 81, 1313-1329. [CrossRef]

30. Termine, E.; Michel, G.P. Transcriptome and secretome analyses of the adaptive response of Pseudomonas aeruginosa to suboptimal growth temperature. Int. Microbiol. 2009, 12, 7-12.

31. Redfern, J.; Wallace, J.; van Belkum, A.; Jaillard, M.; Whittard, E.; Ragupathy, R.; Verran, J.; Kelly, P.; Enright, M.C. Biofilm Associated Genotypes of Multidrug-Resistant Pseudomonas aeruginosa. bioRxiv 2019, 713453. [CrossRef]

32. Tsai, C.J.; Loh, J.M.; Proft, T. Galleria mellonella infection models for the study of bacterial diseases and for antimicrobial drug testing. Virulence 2016, 7, 214-229. [CrossRef]

33. Raetz, C.R.; Reynolds, C.M.; Trent, M.S.; Bishop, R.E. Lipid A modification systems in gram-negative bacteria. Annu. Rev. Biochem. 2007, 76, 295-329. [CrossRef] [PubMed]

34. Steimle, A.; Autenrieth, I.B.; Frick, J.S. Structure and function: Lipid A modifications in commensals and pathogens. Int. J. Med. Microbiol. 2016, 306, 290-301. [CrossRef] [PubMed]

35. Maldonado, R.F.; Sá-Correia, I.; Valvano, M.A. Lipopolysaccharide modification in Gram-negative bacteria during chronic infection. FEMS Microbiol. Rev. 2016, 40, 480-493. [CrossRef] [PubMed]

36. Anandan, A.; Vrielink, A. Structure and function of lipid A-modifying enzymes. Ann. N. Y. Acad. Sci. 2019. [CrossRef]

37. Thaipisuttikul, I.; Hittle, L.E.; Chandra, R.; Zangari, D.; Dixon, C.L.; Garrett, T.A.; Rasko, D.A.; Dasgupta, N.; Moskowitz, S.M.; Malmström, L.; et al. A divergent Pseudomonas aeruginosa palmitoyltransferase essential for cystic fibrosis-specific lipid A. Mol. Microbiol. 2014, 91, 158-174. [CrossRef]

38. Nowicki, E.M.; O’Brien, J.P.; Brodbelt, J.S.; Trent, M.S. Characterization of Pseudomonas aeruginosa LpxT reveals dual positional lipid A kinase activity and co-ordinated control of outer membrane modification. Mol. Microbiol. 2014, 94, 728-741. [CrossRef]

39. Hittle, L.E.; Powell, D.A.; Jones, J.W.; Tofigh, M.; Goodlett, D.R.; Moskowitz, S.M.; Ernst, R.K. Site-specific activity of the acyltransferases HtrB1 and HtrB2 in Pseudomonas aeruginosa lipid A biosynthesis. Pathog. Dis. 2015, 73, ftv053. [CrossRef]

(C) 2019 by the authors. Licensee MDPI, Basel, Switzerland. This article is an open access article distributed under the terms and conditions of the Creative Commons Attribution (CC BY) license (http://creativecommons.org/licenses/by/4.0/). 